\title{
Percepçóes de Risco no Prazer DE Fumar: uma InVESTIGAÇÃo Sob a Ótica do Comportamento Do Consumidor
}

\section{RISK PERCEPTIONS IN TOBACCO CONSUMPTION PLEASURE: AN INVESTIGATION IN THE CONSUMER BEHAVIOR AREA}

MICHELLE HELENA KOVACS Doutoranda em Administração de Empresas pela UFPE/PROPAD. Pesquisadora do COPESQ/FIR (Coordenação de Pó-graduação e Pesquisa) e MKP/UFPE (Centro de Estodos em Mraketing e Pessoas), professora de graduação e de pós-graduação de marketing e comunicação da Faculdade Integrada do Recife - FIR. Rua Setúbal, 860/B - apto. 1103 - Recife - PE - CEP 51030-010

E-mail:kovacs@fir.br

SALOMÃO ALENCAR DE FARIAS Doutor em Administração pela USP. Atualmente cursando o pós-doutorado em Atlanta

(Estados Unidos). Professor pesquisador da Universidade Federal de Pernambuco UFPE/PROPAD e coordenador do Centro de Estudos em Marketing e Pessoas - MKP. Rua Rodrigues de Mendonça, 45 - apto. 1103 - Prado - Recife - PE - CEP 50720-170 E-mail:sał@ufpe.br

\section{CLÁUDIA RAMOS DE OLIVEIRA} Mestra em Administração de empresas pela Universidade Federal do Paraná. Flat 74, Hilltop Court, off Mabfield Road, Wilmslow Road, Fallowfield, Manchester, M14 6 LH. E-mail:croliver10@yahoo.com.br 


\section{RESUMO}

O risco percebido tem sido estudado na área de marketing desde sua introdução, em I960 por Bauer, devido à sua influência no comportamento do consumidor. O objetivo deste estudo foi investigar o construto risco percebido quanto ao consumo de cigarros em grupos de fumantes e não-fumantes, com o intuito de analisar possíveis diferenças de percepções quanto aos tipos de riscos abordados pela literatura especializada. Ademais, buscou-se verificar a propensão a assumir riscos e diferenças quanto à percepção dos benefícios do consumo de cigarros. Para tal, foi realizado um estudo de corte transversal com uma amostra de 390 estudantes universitários. Os resultados indicaram que os fumantes percebem menos riscos de vício, financeiro, de saúde, sociais, de tempo e o risco geral do que os não-fumantes. Os fumantes percebem mais benefícios, em especial quanto considerar o cigarro como uma forma de aliviar a tensão e de ser relaxante.

\section{PALAVRAS-CHAVE}

Comportamento do consumidor; Riscos percebidos; Benefícios percebidos; Tabagismo.

\section{ABSTRACT}

The perceived risk has been studied in the marketing literature since its introduction, in I960 by Bauer, in spite of the importance of this construct in the consumer behavior area. The objective of this study was to investigate the perceived risk construct of the cigarette consumption among smoking and non smoking groups, with the goal to analyze the different perceptions of the risks. Besides that, this investigation studied the differences of perceived benefits of smoking and propensity of taking risks. This investigation used a transversal study with a sample of 390 university students. The results indicated that the smokers perceive less risks of vice, financial, 
health, social, time and the general risk than the non smoking. Besides that, the smokers perceived more benefits, in special considering smoking as a way to alleviate the tension and relax.

\section{KEYWORDS}

Consumer behavior; Perceived risks; Perceived benefits; Tobacco smoking.

\section{INTRODUÇÃ O}

Os malefícios provocados à saúde pelo vício de fumar são amplamente difundidos. Não obstante, o tabagismo é, hoje, a principal causa de enfermidades evitáveis e incapacidades prematuras no mundo (MENEZES e colaboradores, 2002). A cada ano, segundo a Organização Mundial de Saúde (WHO, I999a), morrem cerca de 4 milhões de pessoas em todo o mundo em decorrência de doenças relacionadas ao uso do tabaco. Estima-se que por volta de 2030 , se nada for feito para reduzir a expansão do seu consumo, esse número deva atingir to milhões, sendo que $70 \%$ dessas vítimas estarão concentradas em países em desenvolvimento, a maioria carente de financiamento público para programas sociais (MALCON e colaboradores, 2003).

O que era prerrogativa dos países ricos atinge progressivamente os países em desenvolvimento. $\mathrm{O}$ fenômeno em curso evidencia um reposicionamento da indústria do fumo, que, aliado ao processo de globalização, tem conseguido abrir novos mercados ao mesmo tempo em que mantém os velhos nichos (MENEZES et al., 2002). Segundo estudos desenvolvidos pelo Banco Mundial, o uso do tabaco gera uma perda global de US\$200 bilhões por ano, metade dela ocorre em países em desenvolvimento (WHO, I999b; MINISTÉRIO DA SAÚDE, 2003). Tal valor resulta da ponderação de fatores, tais como tratamento das doenças relacionada ao tabaco, morte de cidadãos em idade produtiva, maior índice de aposentadoria precoce, aumento no índice de absenteísmo e menor rendimento produtivo.

Esses indicadores levaram as instituições internacionais de saúde a eleger o combate à epidemia tabágica como um dos maiores desafios à saúde publica no mundo (WHO, I999a). No Brasil, de acordo com o Ministério da Saúde, um terço da população adulta fuma, proporção considerada alta quando comparada com os outros países da América Latina. Segundo o Instituto Nacional de Câncer (Inca), órgão responsável, no âmbito do governo federal, pelas ações e estudos relativos ao controle do tabaco, são cerca de II,2 milhões de mulheres e i6,7 milhões de homens fumantes; estimam-se aproximadamente 200.000 mortes/ano (MINISTÉRIO DA SAÚDE, 2003). 
No sentido de reverter esse fato, o Ministério da Saúde, por meio do INCA, vem concentrando cada vez mais esforços no combate ao tabagismo, um dos principais fatores de risco para diferentes tipos de câncer. Assim, dentro do Programa Nacional de Controle do Tabagismo foram adotadas ações como a redução dos níveis de substâncias tóxicas presentes nos cigarros nacionais ou importados, obrigatoriedade de estampar nas embalagens imagens ilustrativas sobre os males do fumo e proibição da propaganda de cigarros nos meios de comunicação. Recentemente o governo aprovou uma medida que proíbe as empresas que fabricam cigarros de patrocinar eventos culturais e esportivos, tornando-se um dos países mais severos no combate ao tabagismo (MINISTÉRIO DA SAÚDE, 2003).

Os esforços antitabágicos, no entanto, encontram grandes dificuldades, particularmente em decorrência da estratégia utilizada pela indústria do tabaco: explorar o público jovem. Seduzir e fidelizar os jovens é uma forma de as companhias criarem uma "reserva de reabastecimento", capaz de suprir a demanda atual, que poderia ser ameaçada por aqueles que deixam de fumar ou morrem devido ao fumo, bem como assegurar consumidores regulares amanhã. Aproveita-se, dessa forma, a suscetibilidade do jovem, que nesta fase vivencia a incorporação de hábitos e comportamentos que deverão fazer parte da sua vida adulta.

Nessa guerra, parece que os prazeres supostamente proporcionados pelo hábito de fumar, vendidos pelas mensagens publicitárias, têm conseguido se sobrepor aos riscos reais associados ao consumo do tabaco e freqüentemente exibidos pelas medidas e campanhas desenvolvidas pelos governos e pelas instituições internacionais de saúde ao redor do mundo. Todavia, apesar dos riscos reais existentes quanto ao consumo de cigarros, pouco se sabe quanto aos riscos percebidos pelos fumantes e não-fumantes. Existe uma carência de estudos que analisem o risco percebido, sendo considerado um construto multidimensional, que pode levar a um indivíduo subestimar um risco objetivo ou real. A maior parte dos estudos que analisam o consumo de cigarros é da área médica, em que existe uma vasta literatura sobre o tabagismo, notadamente quanto aos danos causados devido ao consumo de cigarros.

Assim, na área de comportamento do consumidor, em especial no campo de conhecimento sobre riscos percebidos, existe uma carência de estudos que tenham como foco o consumo de cigarros. Devido à importância do tema e à carência de estudos na área, este trabalho teve como objetivo investigar o construto risco percebido quanto ao consumo de cigarros em grupos de fumantes e não-fumantes, com o intuito de analisar possíveis diferenças de percepções quanto aos tipos de riscos abordadas pela literatura especializada. Ademais, buscou-se verificar diferenças entre esses dois grupos quanto à percepção dos benefícios do consumo de cigarros e à propensão a assumir riscos. 


\section{OS RISCOS PERCEBIDOS}

O risco percebido foi introduzido na literatura do Marketing em i960 por Bauer, em seu artigo Consumer behavior as risk-taking. Bauer (I960:24) foi o primeiro pesquisador a propor formalmente que "o comportamento do consumidor envolve risco de forma que qualquer ação do consumidor irá produzir conseqüências na qual ele não poderá antecipar com qualquer aproximação de certeza e na qual algumas são indesejáveis". Corroborando essa idéia, Sitkin e Pablo (I992) afirmam que o risco percebido é considerado como uma característica das decisões nas quais exista a incerteza sobre as conseqüências significantes que possam acontecer. Engel e colaboradores (I995), por sua vez, consideram que o risco vai além da incerteza sobre as conseqüências, sendo uma expectativa pessoal de que uma perda possa ocorrer. Segundo Stem e colaboradores (I977), o risco percebido é uma função dessas incertezas e das possíveis conseqüências. Weber e Bottom (I989) comentam que essas decisões de risco são escolhas entre alternativas, nas quais pelo menos uma das possíveis conseqüências deve ser indesejada, ou menos desejada que as outras, para que o risco exista.

Bauer (I960) salienta que o foco das conseqüências dessas incertezas não seria o risco real (objetivo), e sim o risco percebido (subjetivo). A diferença entre o risco real e o risco percebido é que o risco objetivo existe de fato, contudo pode, ou não, ser percebido pelo consumidor. Por sua vez, o risco subjetivo é o risco que o consumidor percebe e que pode até não existir no campo real, mas apenas na mente do indivíduo, podendo levá-lo a superestimar ou subestimar um determinado risco, visto assim o impacto no comportamento que o risco percebido pode ocasionar (SITKIN e PABLO, I992). Reforçando essa idéia, Schiffman e Kanuk (I997) afirmam que os consumidores são influenciados, apenas, pelo risco que percebem, independentemente do fato de o risco existir realmente, ou não. Assim, o risco que não é percebido, não importando o grau de realidade ou de perigo, não influenciará o comportamento do consumidor.

O risco percebido foi inicialmente definido por Bauer (I960) como um construto bidimensional que envolve as variáveis incertezas e conseqüências. A incerteza seria a probabilidade subjetiva de que um evento ocorra e a conseqüência, o quanto será perdido se as conseqüências do ato não forem favoráveis. A proposta inicial do estudo de Bauer (I960) foi redefinida por uma série de autores (ROSELIUS, I97I; JACOBY e KAPLAN, I972; LUTZ e REILLY, I974; PETER e TARPEY, I975), que propuseram a consideração do risco percebido como um construto multidimensional, incluindo tipos variados de conseqüências. Essas conseqüências foram inicialmente subdivididas em duas categorias por Cunningham (1967): de performance e psicossociais. 
Taylor (1974) considerava o risco percebido por meio de duas categorias: psicossociais e funcionais/econômicas. Alguns pesquisadores utilizaram tais categorias, como Stem e colaboradores (I977), e, gradativamente, outros estudiosos começaram a separar as conseqüências psicossociais em conseqüências psicológicas e sociais, identificando, ainda, outros tipos de conseqüências. Contudo, nem sempre houve um consenso entre os pesquisadores sobre quais seriam os tipos de riscos a serem considerados. Assim, tem sido difícil comparar os resultados obtidos dos estudos de risco percebido.

Roselius (I97I) estudou quatro tipos de perdas: dinheiro, ego, perigo e tempo. Jacoby e Kaplan (I972) conceitualizaram o risco geral em cinco subcomponentes: físico, psicológico, social, financeiro e risco global. Zikmund e Scott (I974) identificaram mais um tipo de risco: a perda de oportunidade futura. Cheron e Ritchie (I982) afirmam que existem sete tipos de risco, acrescentando aos riscos citados anteriormente o risco de satisfação. Lovelock e Wright (I998) estudaram tipos de risco específicos para serviços, nos quais foi acrescentado o risco sensorial, que, de acordo com estes autores, significa qualquer impacto indesejado nos cinco sentidos. Ao estudarem o risco percebido de fumar, Rindfleisch e Crockett (I999) adicionaram, para o caso, o risco percebido do vício. Jenks (I992) concluiu em suas investigações que os fumantes podem perceber mais o risco percebido do vício do que os não-fumantes, devido, de acordo com o autor, aos fumantes usarem o vício como uma forma de redução da dissonância do ato de fumar, ou seja, para racionalizar o seu comportamento como fumante.

Analisando os resultados obtidos nos estudos de risco percebido quanto ao consumo de cigarros, foram escolhidos cinco riscos percebidos para a pesquisa: de vício, saúde, tempo, social e financeiro, que foram os riscos analisados em outros estudos sobre o consumo de cigarros (LOKEN, I982; AITKEN e EADIE, I990; RINDFLEISCH e CROKETT, I999), acrescentando para o caso o risco geral (JACOBY e KAPLAN,I972). Ademais, alguns riscos não se conformam ao estudo em questão como o risco funcional ou o de futura oportunidade perdida.

A seguir são apresentadas as definições dos riscos analisados nesta investigação, com base em estudos prévios sobre o tema, bem como as hipóteses que guiaram a pesquisa.

\section{RISCO DE VÍ́CIO}

Ao estudarem o risco percebido de fumar, alguns autores (JENKS, I992; RINDFLEISCH e CROCKETT, I999) adicionaram, para a literatura da área, o risco percebido do vício. Jenks (I992) concluiu em suas investigações que os fumantes podem perceber mais o risco do vício do que os não-fumantes devido, 
de acordo com o autor, aos fumantes usarem o vício como uma forma de redução da dissonância do ato de fumar, ou seja, para racionalizar o seu comportamento como fumante. Dessa forma, considera-se que o fator risco percebido do risco de vício pode ser um determinante na atitude de uma série de escolhas do comportamento do consumidor, especialmente para as consideradas demandas indesejadas, como o consumo de cigarros. Destarte, levanta-se a primeira hipótese do estudo.

\section{RISCO DE SAÚDE}

Para Solomon (I998), este risco está associado à falta de vigor físico, à saúde e à vitalidade. Segundo Roselius (I97I), alguns produtos são perigosos para a saúde, desenvolvendo o risco percebido em determinados segmentos da população. Supõe-se que fumantes percebam um menor risco de saúde do que nãofumantes, sendo, pois, esta a segunda hipótese da pesquisa:

H2: Os fumantes percebem menos riscos de saúde em relação ao consumo de cigarros do que os não-fumantes.

\section{RISCO DE TEMPO}

De acordo com Engel et al. (1995:313), os recursos do consumidor consistem em dois orçamentos obrigatórios: dinheiro e tempo. Apesar de renda e riqueza serem variáveis críticas, "permitindo a alguns segmentos comprar mais de tudo, contudo não permitem fazer mais de tudo. Fazendo mais coisas requerem um recurso adicional: tempo". Enquanto, teoricamente, o dinheiro não tem limite de expansão, o tempo é restrito. Segundo esses autores, a escassez temporal cria um valor de forma que, para alguns consumidores, "a maior preocupação passa a ser comprar mais tempo do que mais produtos" (ENGEL et al. I995:313). O tempo que um indivíduo perde ao longo de sua vida para consumir este produto foi investigado em outros estudos sobre tabagismo (RINDFLEISCH e CROCKETT, I999). A terceira hipótese desta investigação é então apresentada:

$\mathrm{H}_{3}$ : Os fumantes percebem menos riscos de tempo em relação ao consumo de cigarros do que os não-fumantes. 


\section{RISCO SOCIAL}

De acordo com Solomon (I998), o risco social está associado à auto-estima e autoconfiança e compreende, segundo Lutz e Reilly (I974), a forma como o consumidor pensa que outras pessoas o julgam pelos produtos consumidos. Para Dholakia (I997) o risco social ocorre com a opinião desfavorável ao consumidor por causa do produto ou serviço adquirido. Visto que o tabagismo tem sido, cada vez mais, condenado socialmente, seja por campanhas antitabagistas, seja pela própria postura da sociedade perante o fumante, imagina-se que esses indivíduos percebam um menor risco social do que os não-fumantes.

$\mathrm{H}_{4}$ : Os fumantes percebem menos riscos sociais em relação ao consumo de cigarros do que os não-fumantes.

\section{RISCO FINANCEIRO}

Solomon (i998) afirma que o risco financeiro consiste em qualquer risco relacionado à perda de bens e dinheiro. No estudo realizado por Rindfleisch e Crockett (I999) sobre riscos percebidos em fumar, foi estudada a influência de cinco tipos diferentes de riscos: financeiro, vício, físico, tempo e social. Apesar de os resultados indicarem que os fumantes têm percepções mais baixas para todos os cinco tipos de riscos que os não-fumantes, uma análise multivariada revelou que somente os riscos de vício e financeiro foram significantes. Contudo, entre os jovens adultos que têm tendências a assumir riscos, não houve correlação com o risco do vício. Para o risco financeiro houve uma correlação significante tanto para os jovens adultos com tendências a assumir riscos como para os que têm aversão ao risco. Esse resultado sugere que "as campanhas antitabagistas que focam o risco financeiro podem ser um meio efetivo de desencorajar o fumo tanto entre os caçadores de riscos como aos avessos ao risco" (RINDFLEISCH e CROCKETT, I999:I68). Destarte, é levantada a quinta hipótese desta investigação:

$\mathrm{H}_{5}$ : Os fumantes percebem menos riscos financeiros em relação ao consumo de cigarros do que os não fumantes.

Supõe-se assim que os fumantes percebam menos o risco geral quanto ao consumo de cigarros do que os não-fumantes, sendo esta a sexta hipótese da pesquisa:

H6: Os fumantes percebem menos o risco geral em relação ao consumo de cigarros do que os não-fumantes. 


\section{RISCO INERENTE E \\ RISCO MANIPULADO}

Segundo Bettman (I973), para obter uma maior precisão em modelos que lidam com o risco percebido, é necessária a divisão deste em dois construtos diferentes: o inerente e o manipulado. $\mathrm{O}$ inerente é o risco latente que o consumidor percebe em uma classe de produtos. Por sua vez, o risco manipulado é o nível de conflito dentro de uma classe de produto que o indivíduo percebe ao ter que escolher uma marca entre as outras da mesma classe. $\mathrm{O}$ risco manipulado representa os resultados finais de uma ação de busca de informação no processo de redução do risco inerente. Ou seja, de acordo com o autor, o risco manipulado é o inerente modificado por informação, fidelidade à marca etc. Isso implica o fato de que, quando o consumidor não tem informação alguma, o risco manipulado é igual ao inerente.

Cabe ressaltar que o presente estudo abordou o risco inerente, ou seja, o risco quanto ao tabagismo de uma forma geral, sem especificar uma determinada marca de cigarros. Assim, é possível que para algum segmento desse mercado uma determinada marca possa apresentar menos riscos percebidos do que outra devido à confiança ou a outros fatores que o indivíduo leve em consideração ao analisar as possíveis conseqüências negativas do ato de fumar cigarros dessa marca específica, como menor teor de alcatrão e nicotina.

\section{A PROPENSÃO DE ASSUMIR RISCOS E O CONSUMO DE CIGARROS}

Segundo Sitkin e Pablo (I992), três características individuais são as prováveis determinantes do comportamento de risco: preferências de risco, percepções de risco e propensão ao risco. A preferência por risco é uma característica individual que influencia ações dos indivíduos. De acordo com esses autores, pessoas que gostam do desafio que o risco pode proporcionar estão mais propícias a tomar decisões de risco do que aqueles que não gostam. O segundo determinante é a percepção de risco, definida como a avaliação do indivíduo ante o risco presente em uma situação. A terceira característica individual é a propensão ao risco, conceitualizada mais freqüentemente como uma tendência individual de assumir riscos: seria a tendência de um indivíduo a evitar ou assumir riscos.

De acordo com Engel e colaboradores (I995), os consumidores com tendência a assumir riscos são descritos como os caçadores de riscos. De acordo com Farley (1986), esse segmento pode ser caracterizado pela contínua necessidade de estímulos, bem acima da média da população. Por ficarem entediados mais facilmente, os caçadores de riscos são predispostos a comprar aventuras, são 
mais criativos e extrovertidos. Além disso, citam como objetivos em suas vidas o sucesso e a competência, em contraste com os que evitam correr riscos, que citam a felicidade como objetivo de vida. Cerca de $25 \%$ da população americana faz parte desse segmento (ENGEL e colaboradores, I995:442). Pressupõe-se que a variável propensão a assumir riscos tenha uma influência na relação entre riscos percebidos e o consumo de cigarros, em que os indivíduos que têm tendências a assumir riscos estejam mais dispostos a correr esses riscos do que os indivíduos com uma baixa propensão a assumi-los. Assim, apresenta-se a sétima hipótese do estudo:

H7: Os fumantes têm uma maior propensão a assumir riscos do que os não-fumantes.

\section{A PERCEPÇÃo dos BENEFícios DO CONSUMO DE CIGARROS E OS RISCOS PERCEBIDOS}

Rindfleisch e Crockett (I999) comentam que, apesar dos aspectos negativos envolvidos com o ato de fumar, uma série de fatores positivos é também relacionada ao consumo de cigarros, como: possibilitar o fumante a baixar o peso, ser relaxante, contribuir para a concentração, aliviar a tensão, ser uma experiência prazerosa, entre outros. Esses aspectos podem exercer uma influência na redução do risco percebido pelos indivíduos, como uma forma de minimizar a dissonância cognitiva causada pelo risco percebido em fumar. De acordo com Solomon, I998, e Engel e colaboradores, I995, a dissonância cognitiva pode levar à insatisfação e ao arrependimento da decisão do consumo de um produto. Assim, pressupõe-se que os indivíduos que fumam percebem mais benefícios do que os não-fumantes. Espera-se também que, com base nessas diferenças de percepção dos benefícios do consumo de cigarros, seja possível discriminar grupos de fumantes e não-fumantes:

$\mathrm{H} 8$ : Os fumantes percebem mais benefícios quanto ao consumo de cigarros do que os não-fumantes.

H9: Os benefícios percebidos quanto ao consumo de cigarros podem discriminar grupos de fumantes e não-fumantes.

\section{METODOLOGIA DO ESTUDO}

A pesquisa que originou este artigo foi de natureza descritiva. Devido ao objetivo do trabalho, foi escolhido o estudo do tipo corte transversal, que utiliza a amostra uma única vez, descrevendo o cenário naquele momento (CHURCHILL, I999; MALHOTRA, I993). 
O estudo foi conduzido em duas fases. Na primeira fase, com ênfase qualitativa, houve inicialmente o levantamento de dados secundários. Foram analisados bancos de dados (como o da Organização Mundial de Saúde), estudos previamente publicados sobre o tema por meio de pesquisas bibliográficas em artigos de congressos, periódicos e livros especializados, bem como pela Internet, e, com base nestes, foi elaborado um roteiro de entrevistas semi-estruturado aplicado pelos autores. Ademais, os autores fundamentaram o construto risco percebido por meio da revisão da literatura. Essa fase da pesquisa objetivou verificar aspectos relevantes dos riscos percebidos relacionados ao tabagismo, identificando que tipos de riscos são citados, obtendo um entendimento inicial sobre a diferença de percepção entre os fumantes e não-fumantes.

Para a amostra qualitativa, o número de entrevistas pessoais não foi considerado fixo. Segundo Parasuraman (I986), os pesquisadores da área do comportamento do consumidor não devem se satisfazer com um número baixo de entrevistas, mas deve-se realizá-las até o momento em que se perceba que as novas informações obtidas estejam convergindo para um mesmo conjunto de indicadores. Foram realizadas dez entrevistas pessoais, com a amostra escolhida por conveniência, sendo a metade da amostra composta por fumantes e a outra metade por não-fumantes, para uma melhor adequação à identificação dos riscos percebidos. Foram analisados quais os riscos percebidos pela amostra quanto ao consumo de cigarros para verificar se seria necessária a inclusão de outros riscos percebidos na investigação (além dos cinco abordados em estudos prévios sobre o tema).

Os entrevistados, seis homens e quatro mulheres, são universitários do curso de Administração de Empresas, com idade entre I8 e 25 anos. As entrevistas foram gravadas com o prévio consentimento dos entrevistados. Posteriormente foram transcritas para a análise de conteúdo, tendo como unidade de mensuração as palavras e as frases, buscando identificar tipos de riscos e benefícios percebidos quanto ao consumo de cigarros pela amostra.

Com base nas informações obtidas na fase qualitativa, foi elaborado um instrumento de coleta de dados que foi aplicado a um número maior de respondentes. Nessa segunda fase, agora com ênfase quantitativa, o questionário foi aplicado a uma amostra de 390 alunos universitários. O instrumento foi empregado em salas de aula de uma faculdade da cidade de Recife, para os alunos do curso de Administração de Empresas, Relações Internacionais e Turismo. O pesquisador, durante o horário de aula, solicitava que os alunos participassem da pesquisa, informando previamente a eles que a participação era voluntária e que todas as respostas seriam tratadas de forma confidencial. Os questionários eram entregues para cada aluno, que preenchia as respostas e, ao término, colocava o instrumento de coleta de dados em um envelope, para que não fosse identificado. Inicialmente 
foram utilizados 30 questionários como amostra piloto, em que o entendimento das questões foi verificado, não necessitando de ajustes no questionário.

O questionário apresentou-se em quatro blocos: riscos percebidos quanto ao tabagismo, propensão do respondente ao assumir riscos, benefícios percebidos quanto ao tabagismo e características demográficas. Para o bloco das perguntas sobre os riscos percebidos, foi utilizada uma contextualização, por meio de um personagem, "João", com o intuito de minimizar a tendenciosidade das respostas, fazendo com que os respondentes analisassem os riscos do consumo de cigarros não como para si mesmos, mas para esse personagem, pela técnica de projeção, com a intenção de obter respostas mais fidedignas. Assim, no enunciado da questão do risco percebido, para ambos os sexos, foi apresentada a seguinte problematização: "João é um universitário de 20 anos de idade. Ele fuma um maço de cigarros por dia há dois anos. Ele diz que ele pretende parar de fumar algum dia, mas não sabe exatamente quando. Ele atualmente não está tendo nenhum problema de saúde. Indique nas afirmações abaixo o risco que você acha que ele corre".

No estudo de Rindfleisch e Crockett (I999), os autores também utilizaram uma contextualização para a questão do risco percebido, introduzindo a pergunta com uma figura masculina, "John", para os respondentes do sexo masculino, e uma figura feminina, "Mary", para as respondentes do sexo feminino. Contudo, esses autores não utilizaram, dentro do risco de saúde, a questão da associação do fumo com a impotência sexual, item esse acrescentado no presente estudo, devido à análise de conteúdo das entrevistas pessoais.

A questão foi composta por itens sobre os cinco riscos percebidos encontrados na literatura específica sobre o consumo de cigarros (LOKEN, I982; AITKEN e EADIE, I990; RINDFLEISCH e CROKETT, I999) e estes foram corroborados por meio das entrevistas pessoais da fase exploratória do estudo. Para cada tipo de risco foram elaboradas cinco frases em que os respondentes indicavam o quanto concordavam ou discordavam da existência desses riscos com o consumo de cigarros, finalizando com o risco geral, última questão deste bloco, totalizando 26 itens. Foi utilizada uma escala de cinco pontos, no formato Likert, permitindo aos respondentes atribuir pontos às diferentes categorias de resposta, por meio de uma escala artificial que varia de i a 5, em que i é concordo totalmente, 2 é concordo parcialmente, 3 é nem concordo/nem discordo, 4 é discordo parcialmente e 5 é discordo totalmente (ROESCH, I999; AAKER, I990).

As assertivas sobre os diferentes riscos foram posicionadas de forma que não ficassem agrupadas de acordo com o mesmo tipo de risco. Os usuários não foram informados sobre que tipos de riscos estavam sendo analisados. O Quadro I apresenta as frases utilizadas no questionário para mensurar os riscos percebidos: 


\section{QUADRO I}

\section{ITENS UTILIZADOS NA MENSURAÇAO DOS RISCOS PERCEBIDOS QUANTO AO CONSUMO DE CIGARROS}

\section{Riscos de vício}

1.1 Desenvolver outros comportamentos de vício, como o alcoolismo

1.2 Ficar psicologicamente viciado em cigarros

1.3 Ficar com crise de abstinência (como ansiedade/nervosismo) devido ao vício de fumar

1.4 Sentir-se mal sobre si mesmo, por continuar a fumar

1.5 Ficar biologicamente/quimicamente viciado em cigarros

\section{Riscos financeiros}

2.1 Deixar de assegurar o seu futuro financeiro (como poupança ou previdência privada) ao gastar dinheiro com cigarros

2.2 Gastar muito dinheiro posteriormente com tratamentos de saúde

2.3 Ter que pedir dinheiro emprestado para comprar cigarros

2.4 Não ter dinheiro extra por conta de seu vício de fumar

2.5 Deixar de comprar alimentos ou outros insumos fundamentais para comprar cigarros

\section{Riscos de saúde}

3.1 Desenvolver câncer de pulmão

3.2 Desenvolver uma doença do coração, como hipertensão e infarto

3.3 Desenvolver um enfisema pulmonar e bronquite crônica

3.4 Ficar com impotência sexual

3.5 Desenvolver outros tipos de câncer (boca, bexiga, laringe)

\section{Riscos sociais}

4.1 Causar uma má impressão ao ter um encontro afetivo com uma pessoa que não fume

4.2 Deixar os amigos dele irritados por fumar em suas casas ou carro

4.3 Irritar os pais dele quando ele acende um cigarro

4.4 Incomodar as pessoas com o mau hálito dele, devido ao fumo

4.5 Ser um mau exemplo de comportamento para a sociedade devido ao seu vício

\section{Riscos de tempo}

5.1 Perder tempo de estudo ao parar para fumar

5.2 Perder tempo fumando que ele podia gastar com outras atividades de lazer

5.3 Chegar atrasado na sala de aula porque ele está fumando

5.4 Perder muito tempo tendo que sair dos lugares para fumar

5.5 Perder tempo de vida por conta da redução da sua expectativa de vida

\section{Risco geral: de maneira geral, há riscos para João ao consumir cigarros}

Para a questão da propensão do indivíduo a assumir riscos foi utilizada a mesma escala de cinco pontos do estudo de Rindfleisch e Crockett (I999), que incluiu os seguintes itens: eu gosto de assumir riscos, eu gosto de fazer coisas que as pessoas me dizem que eu não deveria fazer, eu faço coisas que parecem incomodar outras 
pessoas, eu faço coisas que me fazem feliz agora, mas eu posso me arrepender posteriormente, e para me divertir eu sinto que vale a pena entrar em problemas.

Na questão dos benefícios percebidos com o consumo de cigarros foi utilizada a escala de Loken (I982), aplicada em estudos similares sobre o tema, que foi adaptada pelas informações obtidas nas entrevistas pessoais. Foram incluídos cinco itens: fumar ajuda a baixar o peso, alivia a tensão, é uma experiência de sabor prazerosa, é relaxante, ajuda a pessoa a se concentrar.

As variáveis sociodemográficas utilizadas para traçar o perfil dos respondentes foram: sexo, idade e renda familiar. Para a análise desses dados foram utilizadas técnicas de estatística descritiva: freqüência absoluta e percentual e média, desvio padrão, mínimo e máximo das variáveis numéricas.

No que se refere à análise dos dados, para as questões sobre o risco percebido e propensão a assumir riscos foi utilizado o teste t-Student (ALTMAN, I99I); para comparar as médias entre os dois grupos (fumantes e não-fumante) em relação a cada um dos riscos percebidos, propensão a assumir riscos e benefícios percebidos foi utilizado o teste paramétrico $t$-Student, escolhido em função do tamanho da amostra, mesmo considerando a escala de medidas ordinal. Também foi realizada a técnica de análise discriminante considerando-se os riscos percebidos, a propensão a assumir riscos e os benefícios do consumo de cigarros. O nível de significância utilizado foi de $5 \%$. Os dados foram analisados utilizando os softwares estatísticos SPSS na versão II e SAS (Statistical Analysis System) na versão 8.0.

\section{ANÁlise e discussão dOS RESULtAdOS}

A amostra foi composta por $58,4 \%$ respondentes do sexo masculino e $4 \mathrm{I}, 6 \%$ do sexo feminino. Devido à escolha da amostra, incluindo apenas universitários, a maioria dos indivíduos $(85, \mathrm{I} \%)$ se encontrava na faixa etária de I8 a 25 anos. A maior parte da amostra $(6 \mathrm{I}, 6 \%)$ tinha uma renda familiar mensal superior a 20 salários mínimos. Dos 390 pesquisados, 59 (I5,I\%) eram fumantes na data da pesquisa. Portanto, a maioria da amostra foi composta de não-fumantes. Quanto ao número de cigarros consumidos por semana, $28,8 \%$ fumavam mais de 40 cigarros por semana, $27, \mathrm{I} \%$, de I a Io cigarros por semana e $20,4 \%$, de ir a 20 cigarros por semana.

A análise comparativa entre fumantes e não fumantes quanto aos riscos percebidos é apresentada na Tabela I. Exceto para os itens "enfisema pulmonar", "chegar atrasado" e "redução do tempo de vida" existe diferença significativa entre o grupo dos fumantes e dos não-fumantes para todos os outros itens $(\mathrm{P}<0,05$, considerando um nível de significância de 5,0\%, por meio do teste de t-Student). 
TABELA I

RISCOS PERCEBIDOS QUANTO AO CONSUMO DE CIGARROS PELOS GRUPOS DE FUMANTES E NÃO-FUMANTES

\begin{tabular}{|c|c|c|c|c|c|}
\hline \multirow{2}{*}{ Riscos Percebidos } & \multicolumn{2}{|c|}{ Fumantes } & \multicolumn{2}{|c|}{ Não-fumantes } & \multirow{2}{*}{$\mathbf{P}(2)$} \\
\hline & Média & D.P.(1) & Média & D. P. (1) & \\
\hline \multirow{6}{*}{$\begin{array}{l}\text { 1. Vício } \\
\text { 1.1 Desenvolver outros vícios } \\
\text { 1.2 Psicologicamente viciado } \\
\text { 1.3 Crise de abstinência } \\
\text { 1.4 Sentir-se mal sobre si mesmo } \\
\text { 1.5 Bio/quimicamente viciado }\end{array}$} & & & & & \\
\hline & 3,4138 & 1,5676 & 2,6079 & 1,3842 & $0,006\left(^{*}\right)$ \\
\hline & 1,7458 & 1,0922 & 1,3963 & 0,8061 & $<0,001\left(^{*}\right)$ \\
\hline & 2,0678 & 1,1427 & 1,4091 & 0,7474 & $<0,001\left(^{*}\right)$ \\
\hline & 2,1525 & 1,2974 & 1,8800 & 1,0005 & $<0,001\left(^{*}\right)$ \\
\hline & 1,5424 & 1,0225 & 1,3140 & 0,7872 & $<0,001\left(^{*}\right)$ \\
\hline \multicolumn{6}{|l|}{ 2. Financeiro } \\
\hline 2.1 Deixar assegurar futuro & 3,7458 & 1,3721 & 3,1903 & 1,4217 & $<0,001\left(^{*}\right)$ \\
\hline 2.2 Gastar tratamento saúde & 2,0847 & 1,1639 & 1,5485 & 0,8747 & $0,007\left(^{*}\right)$ \\
\hline 2.3 Pedir dinheiro emprestado & 3,0000 & 1,5086 & 2,1843 & 1,2133 & $<0,001\left(^{*}\right)$ \\
\hline 2.4 Não ter dinheiro extra & 3,3621 & 1,5183 & 2,4653 & 1,1682 & $<0,001\left(^{*}\right)$ \\
\hline 2.5 Deixar de comprar insumos & 3,2034 & 1,6377 & 2,4134 & 1,3455 & $0,006\left(^{*}\right)$ \\
\hline \multicolumn{6}{|l|}{ 3. Saúde } \\
\hline 3.1 Câncer de pulmão & 1,4576 & 0,8968 & 1,1398 & 0,5780 & $0,032\left(^{*}\right)$ \\
\hline 3.2 Doença do coração & 1,6271 & 0,8283 & 1,3384 & 0,7469 & $0,001\left(^{*}\right)$ \\
\hline 3.3 Enfisema pulmonar & 1,6271 & 0,9450 & 1,2447 & 0,6353 & 0,1897 \\
\hline 3.4 Impotência sexual & 2,7797 & 1,4747 & 1,9424 & 1,0254 & $<0,001\left(^{*}\right)$ \\
\hline 3.5 Outros tipos de câncer & 1,7119 & 1,0836 & 1,3787 & 0,8058 & $<0,001\left(^{*}\right)$ \\
\hline \multicolumn{6}{|l|}{ 4. Sociais } \\
\hline 4.1 Má impressão & 2,1356 & 1,0741 & 1,8278 & 0,9988 & $<0,001\left(^{*}\right)$ \\
\hline 4.2 Amigos irritados & 2,4746 & 1,3436 & 1,9362 & 1,1094 & $<0,001\left(^{*}\right)$ \\
\hline 4.3 Irritar os pais & 2,1724 & 1,3526 & 1,9757 & 0,9843 & $0,012\left(^{*}\right)$ \\
\hline 4.4 Mau hálito & 1,9492 & 1,1511 & 1,4773 & 0,8920 & $<0,001\left(^{*}\right)$ \\
\hline 4.5 Ser mau exemplo & 3,3621 & 1,4594 & 2,4207 & 1,2634 & $<0,001\left(^{*}\right)$ \\
\hline \multicolumn{6}{|l|}{ 5. Tempo } \\
\hline 5.1 Tempo de estudo & 3,6842 & 1,3648 & 2,6860 & 1,3777 & $0,004\left(^{*}\right)$ \\
\hline 5.2 Atividades de lazer & 3,3390 & 1,4457 & 2,1269 & 1,1866 & $<0,001\left(^{*}\right)$ \\
\hline 5.3 Chegar atrasado & 2,7627 & 1,5238 & 2,2997 & 1,2686 & 0,067 \\
\hline 5.4 Sair dos lugares & 3,2281 & 1,4518 & 2,0816 & 1,1238 & $<0,001\left(^{*}\right)$ \\
\hline 5.5 Redução do tempo de vida & 2,3220 & 1,3827 & 1,7825 & 1,0274 & 0,121 \\
\hline 6. Risco Geral & 1,2712 & 0,5196 & 1,1511 & 0,5517 & \\
\hline
\end{tabular}

(1) D.P. = Desvio padrão.

(2) Probabilidade de significância do teste de t-Student.

(*)Significante ao nível de 5\%, $P<0,05$.

Amostra: fumantes: $n=59$, não-fumantes: $n=331$.

Fonte: banco de dados da pesquisa (2003). 
Visto que o valor 5 significava discordo totalmente e I concordo totalmente, quanto menor o valor indicado, maior o risco percebido. Assim, pode-se observar que, para todos os riscos analisados na pesquisa, a média do grupo dos nãofumantes foi menor do que a média do grupo dos fumantes, em que, para os cinco riscos pesquisados mais o risco geral, os indivíduos fumantes perceberam menos riscos do que os não-fumantes.

Observa-se também que o risco geral e o item "desenvolver câncer de pulmão" foram os riscos com um menor escore, indicando um maior risco percebido para os dois grupos analisados. O item ficar "biologicamente/quimicamente viciado em cigarros" ficou em terceiro lugar dos riscos mais percebidos pelo grupo dos fumantes e o risco de desenvolver um enfisema pulmonar e bronquite crônica para o grupo dos não-fumantes.

A análise comparativa entre fumantes e não-fumantes com respeito à valorização das perguntas relacionadas com a propensão a assumir riscos está apresentada na Tabela 2. Baseando-se num nível de significância de 5,0\%, o teste tStudent mostra que não existe diferença significativa entre as valorizações dos dois grupos (fumantes e não-fumantes) com respeito a cada uma das variáveis: "eu gosto de assumir riscos", "eu gosto de fazer coisas que as pessoas me dizem que eu não deveria fazer" e "para me divertir eu sinto que vale a pena entrar em problemas" ( $\mathrm{P}>0,05)$. Todavia, o teste mostra que existe diferença significativa entre as valorizações dos dois grupos com respeito a cada uma das variáveis "eu faço coisas que parecem incomodar outras pessoas" e "eu faço coisas que me fazem feliz agora, mas eu posso me arrepender posteriormente". Para essas duas perguntas, os não-fumantes atribuíram pontuações maiores que os fumantes (veja as médias correspondentes a cada um desses itens).

\section{TABELA 2}

PROPENSÄO A ASSUMIR RISCOS PELOS GRUPOS DOS FUMANTES E NÃO-FUMANTES

\begin{tabular}{l|c|c|c|c|r}
\hline \multirow{2}{*}{$\begin{array}{c}\text { Propensão a assumir } \\
\text { riscos }\end{array}$} & \multicolumn{2}{c|}{ Fumantes } & \multicolumn{2}{c|}{ Não-fumantes } & \multirow{2}{*}{ P(2) } \\
\cline { 2 - 5 } & Média & D.P.(1) & Média & D. P.(1) & \\
\hline $\mathbf{1}$ Gosto de assumir riscos & 2,7966 & 1,4477 & 2,9015 & 1,4151 & 0,602 \\
$\mathbf{2}$ Pessoas dizem p/ não fazer & 4,0000 & 1,2999 & 3,8845 & 1,2489 & 0,517 \\
$\mathbf{3}$ Incomodar outras pessoas & 3,8136 & 1,3955 & 4,2462 & 1,0579 & $0,006\left(^{*}\right)$ \\
$\mathbf{4}$ Arrepender posteriormente & 2,0339 & 1,2590 & 2,9787 & 1,5190 & $<0,001\left(^{*}\right)$ \\
$\mathbf{5}$ Divertir entrar em prob. & 4,4576 & 0,9882 & 4,4130 & 1,0824 & 0,709 \\
\hline
\end{tabular}

(1) D.P. = Desvio padrão.

$\left.{ }^{*}\right)$ Significante ao nível de $5 \%, P<0,05$. Amostra: fumantes: $n=59$, não fumantes: $n=331$.
(2) Probabilidade de significância do teste de t-Student. Fonte: banco de dados da pesquisa (2003). 
É interessante observar o item "eu faço coisas que me fazem feliz agora, mas eu posso me arrepender posteriormente", em que houve uma diferença estatisticamente significativa entre os dois grupos ( $\mathrm{P}<\mathrm{0}, \mathrm{O} 0 \mathrm{OI})$. Os fumantes, além de perceberem menos riscos quanto ao ato de fumar, podem ter uma maior propensão a assumir riscos, levando ao consumo de cigarros, devido ao prazer proporcionado pelo ato de fumar, mesmo tendo conseqüências futuras. Ou seja, para aproveitarem o presente, parecem estar dispostos a comprometer o futuro. Levanta-se também a hipótese de que os fumantes têm um comportamento mais hedonista, contudo uma menor preocupação com as conseqüências futuras dos seus presentes atos. Ademais, quanto ao segundo item em que houve associação estatisticamente significativa, de fazer coisas que parecem incomodar outras pessoas $(\mathrm{P}<0,05)$, pode ser que os fumantes tenham uma menor percepção dos riscos sociais; segundo Dholakia (I997), o risco social está presente quando o indivíduo está preocupado com a opinião desfavorável de terceiros por conta de produtos adquiridos. Assim, pressupõe-se que o grupo dos fumantes tenha uma maior confiança em seus atos, não levando tanto em consideração a opinião de outros indivíduos, mesmo que suas ações pareçam incomodar outras pessoas.

Quanto aos benefícios percebidos em relação ao consumo de cigarros, uma análise para os dois grupos indicara que os itens: "fumar alivia a tensão" e "ser relaxante" são os benefícios mais percebidos pelos fumantes. Para todos os benefícios percebidos, conforme pode ser observado na Tabela 3, houve uma diferença significativa entre os grupos, em que, para todos os itens analisados, os indivíduos que fumam perceberam mais benefícios do que os não-fumantes.

\section{TABELA 3}

BENEFICIOS PERCEBIDOS QUANTO AO CONSUMO DE CIGARROS

\begin{tabular}{|c|c|c|c|c|c|}
\hline \multirow[t]{2}{*}{ Benefícios percebidos } & \multicolumn{2}{|c|}{ Fumantes } & \multicolumn{2}{|c|}{ Não-fumantes } & \multirow{2}{*}{$\mathbf{P}(2)$} \\
\hline & Média & D.P.(1) & Média & D. P. (1) & \\
\hline Fumar ajuda a baixar o peso & 3,2881 & 1,5980 & 4,1560 & 1,2667 & $<0,001\left(^{*}\right)$ \\
\hline Fumar alivia a tensão & 1,8305 & 1,1165 & 3,8807 & 1,3661 & $<0,001\left(^{*}\right)$ \\
\hline $\begin{array}{l}\text { Fumar é uma experiência } \\
\text { de sabor prazerosa }\end{array}$ & 2,5932 & 1,5100 & 4,3761 & 1,0835 & $<0,001\left(^{*}\right)$ \\
\hline Fumar é relaxante & 1,6780 & 1,0412 & 4,000 & 1,3318 & $<0,001\left(^{*}\right)$ \\
\hline $\begin{array}{l}\text { Fumar ajuda a pessoa } \\
\text { a se concentrar }\end{array}$ & 3,7119 & 1,4862 & 4,5518 & 0,8587 & $<0,001\left(^{*}\right)$ \\
\hline
\end{tabular}

(1) D.P. = Desvio padrão.

(2) Probabilidade de significância do teste de t-Student. ( ${ }^{*}$ Significante ao nível de $5 \%, P<0,05$. Amostra: fumantes: $n=59$, não-fumantes: $n=331$.

Fonte: banco de dados da pesquisa (2003). 
Foi também realizada uma análise discriminante da amostra entre os grupos de fumantes e de não-fumantes com relação aos riscos, benefícios e propensão a assumir riscos de fumar. Inicialmente, foi processado, por meio do software SAS (Statistical Analysis System), o estudo para sete conjuntos de dados (Tabela 4) para identificar qual o grupo de variáveis fornece a maior probabilidade de um individuo selecionado aleatoriamente da população em estudo ser classificado corretamente e que também contemple baixos riscos (admissíveis) de má classificação em cada grupo.

Para cada conjunto foi realizado o teste Box's-M para verificar a hipótese nula de igualdade das matrizes de covariâncias. Em todas as situações o teste revelou que existe diferença significativa entre as matrizes com $\mathrm{P}<0,00$ e por esse motivo, em todas as situações, foi utilizada a função discriminante quadrática. Os resultados da classificação por essa técnica, para cada conjunto (probabilidades a priori proporcionais aos números de pesquisados em cada categoria foram $\mathrm{P}_{\mathrm{I}}=0$,I5I e $\mathrm{P}_{2}=0,849$ ), se encontram na Tabela 4. Como se observa, o conjunto de variáveis que melhor define as referidas exigências é o que utilizou conjuntamente os riscos percebidos, a propensão a assumir riscos e os benefícios percebidos (Pr.I a Pi.26 e P4.I a P4.5 e P5.I a P5.5), em que 98,2\% dos casos do grupo original são corretamente classificados, ou seja, a razão de erro de má classificação é apenas I,8\%, além disso as probabilidades de erro de má classificação em cada grupo são: 6,8\% de serem classificados como não-fumantes entre os fumantes e de $0,9 \%$ de serem classificados como fumantes entre os não-fumantes.

Com intuito de utilizar uma função discriminante mais simples que a função discriminante quadrática, obteve-se, para esse mesmo grupo satisfatório de variáveis, pelo método da função discriminante linear de Fisher para o caso de matrizes de covariância iguais, ${ }^{(*)}$ que 92,1\% dos casos do grupo original são corretamente classificados, ou seja, a razão de erro de má classificação é de apenas $7,9 \%$, entretanto as probabilidades de erro de má classificação para os grupos fumantes e não-fumantes são de 30,5\% e 3,9\%. O erro de classificação de fumantes $(30,5 \%)$ é muito alto quando comparado com o correspondente erro $(6,8 \%)$ obtido pela função discriminante quadrática. Portanto, o referido Método de Fisher não deve ser usado. Nesta situação, decidiu-se realizar a análise pelo "Método de classificação quadrática". A Tabela 5 mostra a matriz de classificação correspondente à classificação quadrática para o grupo de variáveis selecionado.

Por meio do Teste Lâmbda de Wilks verifica-se que existe diferença significativa entre as médias populacionais dos dois grupos. Isso significa que a função discriminante quadrática, associada ao referido grupo de variáveis, tem significado discriminatório ( $\mathrm{P}<\mathrm{0.00I})$

De acordo com a regra de classificação quadrática, considerando-se que os custos de má classificação são iguais, se um novo indivíduo selecionado aleato- 
riamente da população em estudo tem um vetor de respostas $X_{\circ}^{\prime}=$ (PI.I a Pi.26 e P4.I a P4-5 e P5.I a P5.5), então ele deve ser classificado no grupo de fumantes:

$$
\frac{-1}{2} X_{0}^{\prime}\left(S^{-1}-S_{2}^{-1}\right) X_{0}+\left(\bar{X}_{1}^{\prime} S_{1}^{-1}-\bar{X}_{2}^{\prime} S_{2}^{-1}\right)-K \geq \ln (P 2 / P 1)
$$

onde

$X_{0}$ é o vetor transposto de $X_{0}$,

$S_{1}^{-1}$ e $S_{2}^{-1}$ são as inversas das matrizes de variâncias e covariâncias dos grupos i e 2

$\bar{X}_{1}{ }^{\prime}$ e $\bar{X}_{2}{ }^{\prime}$ são os vetores de médias para os grupos i e 2

$$
K=\frac{1}{2} \ln \left(\frac{\operatorname{det} S 1}{\operatorname{det} S 2}\right)+\frac{1}{2}\left(X_{1} S_{1}^{-1} \bar{X}_{1}^{\prime \prime}-\bar{X}_{2} S_{2}^{-1} X_{2}^{\prime \prime}\right)
$$

PI e P2 são as prioris utilizadas, que no caso foram as proporções de cada um dos pesquisados classificados em cada grupo det é o determinante.

Ao contrário do método de classificação linear de Fisher (para um novo indivíduo do grupo), cujos cálculos poderiam ser realizados com uma simples calculadora, este método seria bastante trabalhoso, sendo indicado o desenvolvimento de um programa computacional para a sua realização.

\section{TABELA 4}

PERCENTUAL DE CLASSIFICAÇAO INCORRETA EM CADA GRUPO

E PERCENTUAL DE CLASSIFICAÇÃO CORRETA NO GRUPO TOTAL SEGUNDO O CONJUNTO DE DADOS

\begin{tabular}{l|r|r|l}
\hline \multicolumn{1}{c|}{ Conjunto de Variáveis } & $\mathbf{p}^{(1)}$ & $\mathbf{p}^{(2)}$ & $\mathbf{p}^{(3)}$ \\
\hline Riscos percebidos (P.1.1 a P.1.26) & 27,1 & 3,0 & 94,1 \\
Propensão a assumir riscos (P.4.1 a 4.5) & 79,7 & 3,0 & 85,4 \\
Benefícios percebidos (P.5.1 a P.5.5) & 20,3 & 10,3 & 88,2 \\
P.1.1 a P.1.26 e P.4.1 a P.4.5 & 15,3 & 0,9 & 96,9 \\
P.1.1 a P.1.26 e P.5.1 a P.5.5 & 11,9 & 3,3 & 95,4 \\
P.4.1 a P.4.5 e P.5.1 a P.5.5 & 18,6 & 8,5 & 64,4 \\
P.1.1 a P.1.26 e P.4.1 a P.4.5 e P.5.1 a P.5.5 & 6,8 & 0,9 & 98,2 \\
\hline
\end{tabular}

(1) Proporção condicional de ser classificado como fumante entre os não-fumantes.

(2) Proporção condicional de ser classificado como não-fumante entre os fumantes.

(3) Proporção de indivíduos classificados corretamente no grupo total. 


\section{TABELA 5}

\begin{tabular}{|c|c|c|c|c|c|c|}
\hline $\begin{array}{r}\text { PERCE } \\
\text { EM CADA } \\
\text { CORRETA NO } \\
\text { DOS RISC } \\
\text { R }\end{array}$ & $\begin{array}{l}\mathrm{DE} \\
\mathrm{ET} \\
\mathrm{O} \mathrm{T} \\
\mathrm{RCE} \\
\mathrm{E} \mathrm{B}\end{array}$ & $\begin{array}{l}\text { LAS } \\
\text { RCI } \\
\text { TAL } \\
\text { IDO } \\
\text { TEF }\end{array}$ & $\begin{array}{l}\text { IFIC } \\
\text { NTU } \\
\text { PARA } \\
\text { PR } \\
\text { CIOS }\end{array}$ & $\begin{array}{l}\text { ÇAO } \\
\text { DE } \\
\text { CO } \\
\text { ENS } \\
\text { ERC }\end{array}$ & $\begin{array}{l}\mathrm{OOR} \\
\text { SSI } \\
\text { NT } \\
\text { A A } \\
\text { DOS }\end{array}$ & $\begin{array}{l}\text { ADOS } \\
\text { ADOS }\end{array}$ \\
\hline \multirow{3}{*}{ Grupo } & \multicolumn{4}{|c|}{$\begin{array}{c}\text { Classificação pela Análise } \\
\text { de Discriminante }\end{array}$} & \multirow{2}{*}{\multicolumn{2}{|c|}{ TOTAL }} \\
\hline & \multicolumn{2}{|c|}{ Fumantes } & \multicolumn{2}{|c|}{ Não-fumantes } & & \\
\hline & $\mathbf{n}$ & $\%$ & $\mathbf{n}$ & $\%$ & n & $\%$ \\
\hline Fumantes & 55 & 93,2 & 4 & 6,8 & 59 & 100,0 \\
\hline Não fumantes & 3 & 0,9 & 328 & 99,1 & 331 & 100,0 \\
\hline Grupo total & 58 & 14,8 & 332 & 85,1 & 390 & 100,0 \\
\hline
\end{tabular}

Obs.: a proporção de casos corretos na amostra é de $98,2 \%$, correspondendo a $383(55+328)$.

\section{LIMITAÇÕES}

O estudo apresenta diversas limitações, especialmente quanto à generalização dos resultados, que deve ser realizada com cautela. Algumas outras limitações são apresentadas a seguir, cabendo ressaltar que não são exaustivas.

- Apesar de a amostra de adultos jovens (I8 a 25 anos de idade) representar um importante segmento da população fumante brasileira, os esforços das últimas pesquisas têm sido direcionados para os adolescentes, bem como as campanhas antitabagistas para esse público têm recebido atenção especial. A maior parte dos fumantes começou a fumar antes dos I8 anos (PECHMANN e RATNESHWAR, I994). Segundo dados da OMS (Organização Mundial da Saúde), 50\% dos jovens que experimentam derivados do tabaco (cigarro, cigarro de Bali, charuto, cachimbo) se tornam fumantes na vida adulta. Por esse motivo, os adolescentes são um dos alvos dos esforços da OMS para controlar o tabagismo no mundo, pois pesquisas comprovam que $90 \%$ dos adultos fumantes começaram a fumar antes dos ig anos sem perceber os efeitos da nicotina. Em geral, os adolescentes saem da fase de experimentação para a de dependência em um ano ou até menos. De acordo com as informações do Ministério da Saúde (2003), as crianças e os adolescentes estão formando sua identidade, miram-se nos hábitos dos mais velhos e podem ser tentados a imitar os fumantes durante o seu processo de individualização. 
- Apesar de se ter incluído na pesquisa a variável "propensão a assumir riscos", outras variáveis não foram consideradas - como auto-estima, rigidez ou ansiedade (SCHANINGER, I976; COX, I967) - na investigação da influência da personalidade com a percepção de riscos. Para Ross (I975), o risco percebido é uma função de diversas variáveis como diferença de situações e variáveis intrapessoais. Corroborando essa idéia, para Shiffman e Kanuk (I997), a percepção de risco varia não apenas com a personalidade, mas, também, com a situação e cultura. Ademais, Roehl e Fesenmaier (I992) criticam os estudos baseados na premissa de que os indivíduos têm diferentes inclinações a aceitar riscos, entrar em situações perigosas e de que traços de personalidade podem ser usados para explicar o comportamento de risco, pois, segundo eles, grande parte das pesquisas produziu resultados confusos.

\section{SUGESTÕES PARA FUTURAS PESQUISAS}

Sugerem-se analisar os riscos percebidos quanto ao consumo de cigarros utilizando o risco manipulado (BETTMAN, I973), possibilitando a comparação de possíveis diferentes níveis e tipos de riscos percebidos entre as diversas marcas de cigarro, para alguns segmentos de mercado. Por exemplo, talvez as marcas que tenham como apelo comercial o fato de ter um menor teor de alcatrão e nicotina, direcionando seus esforços de comunicação para o público jovem, sejam percebidas por esse segmento com apresentando menor risco de saúde do que as outras marcas.

Ou realizar um estudo com diversas marcas de cigarro (risco manipulado), identificando os diferentes apelos de comunicação utilizados pelas mesmas marcas como status, masculinidade, aceitação social, entre outros, analisando os benefícios percebidos e os riscos percebidos entre elas.

\section{CONCLUSÕES}

Os resultados indicaram que os fumantes percebem menos riscos do que os não-fumantes quanto ao consumo de cigarros, para todos os riscos percebidos analisados: financeiros, de saúde, sociais, de tempo, vício e risco geral. Assim, exceto para três itens, as hipóteses I a 7 foram confirmadas. Destaca-se, entretanto, que, com exceção de dois itens, os demais apresentaram diferenças entre os grupos com valores inferiores a I,০०. O risco geral e o item desenvolver câncer 
de pulmão foram os riscos mais percebidos para ambos os grupos analisados, fumantes e não-fumantes. O item ficar biologicamente/quimicamente viciado em cigarros ficou em terceiro lugar dos riscos mais percebidos pelo grupo dos fumantes e o risco desenvolver um enfisema pulmonar e bronquite crônica, para o grupo dos não-fumantes. Jenks (1992) concluiu em suas investigações que os fumantes podem perceber mais o risco do vício do que os não-fumantes, como uma forma de racionalizar o seu comportamento como fumante. De acordo com Shiffman e Kanuk (I997), os consumidores desenvolvem suas próprias estratégias de redução do risco percebido.

A Hipótese 8, que buscava analisar a propensão de assumir riscos e os grupos fumantes e não-fumantes, não foi confirmada. Para os cinco itens da escala, apenas dois foram estatisticamente significativos, em que indicavam que os fumantes são mais propensos a assumir riscos: "fazer coisas que me fazem feliz agora, mas posso me arrepender posteriormente" e "fazer coisas que parecem incomodar outras pessoas" $(\mathrm{P}<0,05)$, além do mais, a diferença entre as médias dos grupos foi no máximo de 0,94 . Assim, levanta-se a hipótese de que os fumantes não seriam necessariamente mais propensos a assumir riscos, mas teriam uma menor percepção ou sensibilização quanto aos danos envolvidos com o tabagismo ou uma maior percepção dos benefícios quanto o consumo de cigarros.

Esses benefícios percebidos podem ser também uma forma de justificar o comportamento de consumo de cigarros, principalmente quanto à questão de ser relaxante e diminuir o estresse. Para todos os itens analisados quanto aos benefícios do consumo de cigarros, houve diferença estatística significativa entre os dois grupos, sendo todas as diferenças superiores a o,83.

Os resultados da função discriminante quadrática para os dados relativos aos riscos percebidos, propensão ao risco e benefícios percebidos indicaram que $98,2 \%$ dos casos no grupo total foram bem classificados por essa técnica e apenas $6,8 \%$ mal classificados no grupo de fumantes e $0,9 \%$, no grupo de nãofumantes. Assim, as Hipóteses 8 e 9 foram confirmadas no estudo.

Os fumantes podem, apesar de saberem da possibilidade de terem conseqüências negativas futuras, assumir um comportamento de risco, item analisado na propensão de assumir riscos, em que houve uma associação altamente significativa com a assertiva "fazer coisas que me façam feliz agora, mas posso me arrepender posteriormente”. Assim, a sensação proporcionada de relaxamento, causada pelas diversas drogas presentes no cigarro, pode ser uma forma de racionalizar os riscos futuros quanto às conseqüências do consumo de cigarros. Com esse raciocínio, o prazer presente compensaria os possíveis danos futuros. Visto a diferença de percepção desses benefícios, pressupõe-se que ou os fumantes são pessoas que têm um estilo de vida mais tenso e estressante, que sentem necessidade do ato de fumar como uma forma de minimizar, mesmo 
que apenas momentaneamente, esse desconforto psicológico, ou os não-fumantes, mesmo vivendo sob as mesmas circunstâncias, podem, de outras formas, minimizar o estresse, como por meio de esportes, leitura, entre outros.

Assim, faz-se a pergunta se uma campanha que busque sensibilizar os indivíduos quanto a ações que podem ser realizadas para melhorar a qualidade de vida, como prática de exercícios, arte, música, seria aliada estratégicas na concorrência das drogas como uma fonte de "terapia" contra o estresse.

\section{REFERÊNCIAS}

AAKER, David A. Marketing research. 4. ed. Belmont: John Wiley \& Sons, I990.

AITKEN, P.; EADIE, D. Reinforcing effects of cigarette advertising on under-age smoking. British Journal of Addiction, v. 83, n. 3, p. 399-4I2, I990.

ALTMAN, D. G. Practical Statistical for Medical Research. Great Britain. London: Chapman and Hall, I99i. 6ii p.

BAUER, R. Consumer behavior as risk-taking. Proceedings of the $43^{\text {rd }}$. Conference of The American Marketing Association, p. 389-398, I960.

BETTMAN, J. R. Perceived risk and its components: a model and empirical test. Journal of Marketing Research, v. IO, p. I84-I90, may I973.

CHERON, E. J. e RITCHIE, J.R. Leisure activities and perceived risk. Journal of Leisure Research, v. I4, n. 2, p. I39-I54, I982.

CHURCHILL, G. Marketing Research. 7. ed. Orlando: The Dryden Press, I999.

COX, D.F. Risk taking and information handling in consumer behavior. Boston: Harvard University Press, I967.

CUNNINGHAM, S. M. The major dimensions of perceived risk. Boston: Harvard University Press, I967.

DHOLAKIA, U. M. An investigation of the relationship between perceived risk and product involvement. Advances in Consumer Research, v. 24, p. 159-167, I997.

ENGEL, J. F.; BLACKWELL, R. D.; MINIARD, P. W. Consumer Behavior. 8. ed. Orlando: The Dryden Press, I995. 95I p.

FARLEY, F. The big T in personality. Psychology Today, v. 20, p. 44-52, may I986.

JACOBY, J.; KAPLAN, L. The components of perceived risk. In: VENKATESAN, M. Proceedings. Advances in Consumer Research. Chicago University, v. 3, p. 382-393, I972.

JENKS, R. J. Attitudes, perceptions, and risk-taking behaviors of smokers, ex-smokers and nonsmokers. Journal of Social Psychology, n. 5, v. 132, p. 569-575, I992.

LOKEN, B. Heavy smokers', light smokers', and nonsmokers' beliefs about cigarette smoking. Journal of Applied Psychology, n. 5, v. 67, p. 616-622, 1982.

LOVELOCK, C.; WRIGHT, L. Principles of service marketing and management. New Jersey: Prentice Hall, I998.

LUTZ, R. J. e REILLY, P. J. An exploration of the effects of perceived social and performance risk on consumer information acquisition. Advances in Consumer Research, v. I, p. 393-403, I974.

MALCON, M. C., MENEZES, A. M. B.; CHATKIN, M. Prevalência e fatores de risco para tabagismo em adolescentes. Rev. Saúde Pública, v. 37, n. I, p. I-7, fev. 2003. 
MALHOTRA, N. K. Marketing research: an applied orientation. New Jersey: Prentice Hall, I993. 857 p. MENEZES, A. M. B., HORTA, B. L., OLIVEIRA, A. L. B et al. Risco de câncer de pulmão, laringe e esôfago atribuível ao fumo. Rev. Saúde Pública, v. 36, n. 2, p. I29-I34, abr. 2002.

MINISTÉRIO DA SAÚDE. Instituto Nacional de Câncer. Tabagismo. Disponível em: $<$ http:www.inca.gov.br/tabagismo>. Acesso em: I4 de abril de 2003. PARASURAMAN, A. Marketing research. Canada: Addison-Wesley Publishing Company, I986. PECHMANN, C.; RATNESHWAR, S. The effects of antismoking and cigarette advertising on young adolescents perceptions of peers who smoke. The journal of Consumer Research, v. 2I, n. 2, p. 236-25I, sep. I994.

PETER, P. J.; TARPEY, L. X. Behavioral decision making: a comparison of three models. Advances in Consumer Research, v. 2, p. II9-I32, I975.

RINDFLEISCH, A.; CROCKETT, D. Cigarette smoking and perceived risk: a multidimensional investigation. Journal of Public Policy \& Marketing, Ann Arbor, v. I8, n. 2, p. I59-I7I, fall I999.

ROESCH, S. M. A. Projetos de estágio e de pesquisa em administração. 2. ed. São Paulo: Atlas, I999.

ROEHL, W. S.; FESENMAIER, D. R. Risk perceptions and pleasure travel: an exploratory analysis. Journal of Travel Research, Boulder, v. 30, n. 4, p. I7- 22, spring 1992.

ROSELIUS, T. Consumer rankings of risk reduction methods. Journal of Marketing, v. 35, p. 56-6I, jan. I97I.

ROSS, I. Perceived risk and consumer behavior: a critical review. Advances in Consumer Research, v. 2, p. I-20, I975.

SCHANINGER, C. M. Perceived risk and personality. The journal of Consumer Research, v. 3, n. 2, p. 95-IO०, sep., I976.

SCHIFFMAN, L. G. e KANUK, L. L. Comportamento do consumidor. Trad. Vicente Abrósio. 6. ed. Rio de Janeiro: LTC, I997.

SITKIN, S. B. e PABLO, A. L. Reconceptualizing the determinants of risk behavior. The Academy of Management Review, Mississippi State, v. I7, n. I, p. 9-39, jan. I992.

SOLOMON, M.R. Consumer behavior: buying, having and being. 4. ed. New Jersey: Prentice Hall, I998. $640 \mathrm{p}$.

STEM JR., D.E.; LAMB JR., C. W.; MACLACHLAN, D. L. Perceived risk: a synthesis. European Journal of Marketing, Bradford, v. II, n. 4, p. 3I2, I977.

TAYLOR, J. W. The role of risk in consumer behavior. Journal of Marketing, v. 38, p. 54-60, apr. I974.

WEBER, E. U.; BOTTOM, W. P. Axioral measures of perceived risk: some tests and extensions. Journal of Behavioral Decision Making, v. 2, n. 2, p. II3-I3I, I989.

[WHO] WORD HEALTH ORGANIZATION. TOBACCO. Health Facts. Fact Sheet n. 22I, april I999a. Disponível em: <http://who.int/info-fs/fact22I.html>. Acesso em: 4 de abril de 2003.

[WHO] WORD HEALTH ORGANIZATION. TOBACCO. Supporting the Tobacco Industry is Bad Economics. Fact Sheet n. 223, april I999b. Disponível em: <http://who.int/info-fs/fact223.html>. Acesso em: 4 de abril de 2003 .

ZIKMUND, W. G.; SCOTT, J. E. Variety analysis of perceived risk self-confidence and information sources. Advances in Consumer Research, v. I, p. 406-.4I6, I974.

T R A MITAÇ Ã O

Recebido em 17/03/2004

Aprovado em 19/09/2004 
Copyright of Revista de Administração Mackenzie is the property of Universidade Presbiteriana Mackenzie, RAM-Revista de Administracao Mackenzie and its content may not be copied or emailed to multiple sites or posted to a listserv without the copyright holder's express written permission. However, users may print, download, or email articles for individual use. 